\title{
High-Resolution Source Localization Algorithm Based on the Conjugate Gradient
}

\author{
Hichem Semira, ${ }^{1}$ Hocine Belkacemi, ${ }^{2}$ and Sylvie Marcos $^{2}$ \\ ${ }^{1}$ Département d'électronique, Université d'Annaba, BP 12, Sidi Amar, Annaba 23000, Algeria \\ ${ }^{2}$ Laboratoire des Signaux et Systèmes (LSS), CNRS, 3 Rue Joliot-Curie, Plateau du Moulon, 91192 Gif-sur-Yvette Cedex, France
}

Received 28 September 2006; Revised 5 January 2007; Accepted 25 March 2007

Recommended by Nicola Mastronardi

This paper proposes a new algorithm for the direction of arrival (DOA) estimation of $P$ radiating sources. Unlike the classical subspace-based methods, it does not resort to the eigendecomposition of the covariance matrix of the received data. Indeed, the proposed algorithm involves the building of the signal subspace from the residual vectors of the conjugate gradient (CG) method. This approach is based on the same recently developed procedure which uses a noneigenvector basis derived from the auxiliary vectors (AV). The AV basis calculation algorithm is replaced by the residual vectors of the CG algorithm. Then, successive orthogonal gradient vectors are derived to form a basis of the signal subspace. A comprehensive performance comparison of the proposed algorithm with the well-known MUSIC and ESPRIT algorithms and the auxiliary vectors (AV)-based algorithm was conducted. It shows clearly the high performance of the proposed CG-based method in terms of the resolution capability of closely spaced uncorrelated and correlated sources with a small number of snapshots and at low signal-to-noise ratio (SNR).

Copyright (C) 2007 Hichem Semira et al. This is an open access article distributed under the Creative Commons Attribution License, which permits unrestricted use, distribution, and reproduction in any medium, provided the original work is properly cited.

\section{INTRODUCTION}

Array processing deals with the problem of extracting information from signals received simultaneously by an array of sensors. In many fields such as radar, underwater acoustics and geophysics, the information of interest is the direction of arrival (DOA) of waves transmitted from radiating sources and impinging on the sensor array. Over the years, many approaches to the problem of source DOA estimation have been proposed [1]. The subspace-based methods, which resort to the decomposition of the observation space into a noise subspace and a source subspace, have proved to have high-resolution (HR) capabilities and to yield accurate estimates. Among the most famous HR methods are MUSIC [2], ESPRIT [3], MIN-NORM [4], and WSF [5]. The performance of these methods however degrades substantially in the case of closely spaced sources with a small number of snapshots and at a low SNR. These methods resort to the eigendecomposition (ED) of the covariance matrix of the received signals or a singular value decomposition (SVD) of the data matrix to build the signal or noise subspace, which is computationally intensive specially when the dimension of these matrices is large.
The conjugate gradient (CG)-based approaches were initially proposed in the related fields of spectral estimation and direction finding in order to reduce the computational complexity for calculating the signal and noise subspaces. Indeed, previous works [6-8] on adaptive spectral estimation have shown that the modified CG algorithm appears to be the most suitable descent method to iteratively seek the minimum eigenvalue and associated eigenvector of a symmetric matrix. In [8], a modified CG spectral estimation algorithm was presented to solve the constrained minimum eigenvalue problem which can also be extended to solve the generalized eigensystem problem, when the noise covariance matrix is known a priori. In the work of Fu and Dowling [9], the CG method has been used to construct an algorithm to track the dominant eigenpair of a Hermitian matrix and to provide the subspace information needed for adaptive versions of MUSIC and MIN-NORM. In [10], Choi et al.have introduced two alternative methods for DOA estimation. Both techniques use a modified version of the CG method for iteratively finding the weight vector which is orthogonal to the signal subspace. The first method finds the noise eigenvector corresponding to the smallest eigenvalue by minimizing the Rayleigh quotient of the full complex-valued covariance 
matrix. The second one finds a vector which is orthogonal to the signal subspace directly from the signal matrix by computing a set of weights that minimizes the signal power of the array output. Both methods estimate the DOA in the same way as the classical MUSIC estimator. In [11], an adaptive algorithm using the CG with the incorporation of the spatially smoothing matrix has been proposed to estimate the DOA of coherent signals from an adaptive version of Pisarenko. In almost all research works, the CG has been used in a similar way to the ED technique in the sense that the objective is to find the noise eigenvector and to implement any subspacebased method to find the DOA of the radiating sources.

In this paper, the CG algorithm, with its basic version given in [12], is applied to generate a signal subspace basis which is not based on the eigenvectors. This basis is rather generated using the residual vectors of the CG algorithm. Then, using the localization function and rank-collapse criterion of Grover et al. in $[13,14]$, we form a DOA estimator based on the collapse of the rank of an extended signal subspace from $P+1$ to $P$ (where $P$ is the number of sources). This results in a new high-resolution direction finding technique with a good performance in terms of resolution capability for the case of both uncorrelated and correlated closely spaced sources with a small number of snapshots and at low SNR.

The paper is organized as follows. In Section 2, we introduce the data model and the DOA estimation problem. In Section 3, we present the CG algorithm. Our proposed CG-based algorithm for the DOA estimation problem following the same steps in $[13,14]$ is presented in Section 4. After simulations with comparison of the new algorithm to the MUSIC, ESPRIT, and AV-based algorithms in Section 5, a few concluding remarks are drawn in Section 6.

\section{DATA MODEL}

We consider a uniformly spaced linear array having $M$ omnidirectional sensors receiving $P(P<M)$ stationary random signals emanating from uncorrelated or possibly correlated point sources. The received signals are known to be embedded in zero mean spatially white Gaussian noise with unknown variance $\sigma^{2}$, with the signals and the noise being mutually statistically independent. We will assume the signals to be narrow-band with center frequency $v_{0}$. The $k$ th $M$-dimensional vector of the array output can be represented as

$$
\mathbf{x}(k)=\sum_{j=1}^{P} \mathbf{a}\left(\theta_{j}\right) s_{j}(k)+\mathbf{n}(k),
$$

where $s_{j}(k)$ is the $j$ th signal, $\mathbf{n}(k) \in \mathrm{C}^{M \times 1}$ is the additive noise vector, and $\mathbf{a}\left(\theta_{j}\right)$ is the steering of the array toward direction $\theta_{j}$ that is measured relatively to the normal of the array and takes the following form:

$$
\mathbf{a}\left(\theta_{j}\right)=\left[1, e^{j 2 \pi \nu_{0} \tau_{j}}, e^{j 2 \pi 2 \nu_{0} \tau_{j}}, \ldots, e^{j 2 \pi(M-1) \nu_{0} \tau_{j}}\right]^{T}
$$

where $\tau_{j}=(d / c) \sin \left(\theta_{j}\right)$, with $c$ and $d$ designating the signal propagation speed and interelement spacing, respectively. Equation (1) can be rewritten in a compact form as

$$
\mathbf{x}(k)=\mathbf{A}(\boldsymbol{\Theta}) \mathbf{s}(k)+\mathbf{n}(k)
$$

with

$$
\begin{aligned}
\mathbf{A}(\boldsymbol{\Theta}) & =\left[\mathbf{a}\left(\theta_{1}\right), \mathbf{a}\left(\theta_{2}\right), \ldots, \mathbf{a}\left(\theta_{P}\right)\right], \\
\mathbf{s}(k) & =\left[\mathbf{s}_{1}(k), \mathbf{s}_{2}(k), \ldots, \mathbf{s}_{P}(k)\right]^{T},
\end{aligned}
$$

where $\boldsymbol{\Theta}=\left[\theta_{1}, \theta_{2}, \ldots, \theta_{P}\right]$. We can now form the covariance matrix of the received signals of dimension $M \times M$

$$
\mathbf{R}=\mathbf{E}\left[\mathbf{x}(k) \mathbf{x}^{H}(k)\right]=\mathbf{A}(\boldsymbol{\Theta}) \mathbf{R}_{s} \mathbf{A}(\boldsymbol{\Theta})^{H}+\sigma^{2} \mathbf{I},
$$

where $(\cdot)^{H}$ and I denote the transpose conjugate and the $M \times M$ identity matrix, respectively. $\mathbf{R}_{s}=E\left[\mathbf{s}(t) \mathbf{s}^{H}(t)\right]$ is the signal covariance matrix, it is in general a diagonal matrix when the sources are uncorrelated and is nondiagonal and possibly singular for partially correlated sources. In practice, the data covariance matrix $\mathbf{R}$ is not available but a maximum likelihood estimate $\widehat{\mathbf{R}}$ based on a finite number $K$ of data samples can be used and is given by

$$
\widehat{\mathbf{R}}=\frac{1}{K} \sum_{k=1}^{K} \mathbf{x}(k) \mathbf{x}^{H}(k) .
$$

\section{CONJUGATE GRADIENT (CG) ALGORITHM}

The method of conjugate gradients (CG) is an iterative inversion technique for the solution of symmetric positive definite linear systems. Consider the Wiener-Hopf equation

$$
\mathbf{R} \mathbf{w}=\mathbf{b}
$$

where $\mathbf{R} \in \mathcal{C}^{M \times M}$ is symmetric positive definite. There are several ways to derive the CG method. We here consider the approach from [12] which minimizes the following cost function:

$$
\Phi(\mathbf{w})=\mathbf{w}^{H} \mathbf{R} \mathbf{w}-2 \operatorname{Re}\left(\mathbf{b}^{H} \mathbf{w}\right) .
$$

Algorithm 1 depicts a basic version of the CG algorithm. $\alpha_{i}$ is the step size that minimizes the cost function $\Phi(\mathbf{w}), \beta_{i}$ provides $\mathbf{R}$-orthogonality for the direction vector $\mathbf{d}_{i}, \mathbf{g}_{i}$ is the residual vector defined as

$$
\mathbf{g}_{i}=\mathbf{b}-\mathbf{R} \mathbf{w}_{i}=-\nabla \Phi\left(\mathbf{w}_{i}\right)
$$

with $\nabla(\Phi)$ denoting the gradient of function $\Phi$ and $i$ denoting the CG iteration.

After $D$ iterations of the conjugate gradient algorithm the set of search directions $\left\{\mathbf{d}_{1}, \mathbf{d}_{2}, \ldots, \mathbf{d}_{D}\right\}$ and the set of gradients (residuals) $\mathbf{G}_{\mathrm{cg}, D}=\left\{\mathbf{g}_{\mathrm{cg}, 0}, \mathbf{g}_{\mathrm{cg}, 1}, \ldots, \mathbf{g}_{\mathrm{cg}, D-1}\right\}$ have some 


$$
\begin{aligned}
\mathbf{w}_{0}=0, \mathbf{d}_{1}=\mathbf{g}_{\mathrm{cg}, 0}=\mathbf{b}, \rho_{0}=\mathbf{g}_{\mathrm{cg}, 0}^{H} \mathbf{g}_{\mathrm{cg}, 0} \\
\text { for } i=1 \text { to } D \text { do } \\
\mathbf{v}_{i}=\mathbf{R d}_{i} \\
\alpha_{i}=\frac{\rho_{i-1}}{\mathbf{d}_{i}^{H} \mathbf{v}_{i}} \\
\mathbf{w}_{i}=\mathbf{w}_{i-1}+\alpha_{i} \mathbf{d}_{i} \\
\mathbf{g}_{\mathrm{cg}, i}=\mathbf{g}_{\mathrm{cg}, i-1}-\alpha_{i} \mathbf{v}_{i} \\
\rho_{i}=\mathbf{g}_{\mathrm{cg}, i}^{H} \mathbf{g}_{\mathrm{cg}, i} \\
\beta_{i}=\frac{\rho_{i}}{\rho_{i-1}}=\frac{\left\|\mathbf{g}_{\mathrm{cg}, i}\right\|^{2}}{\left\|\mathbf{g}_{\mathrm{cg}, i-1}\right\|^{2}} \\
\mathbf{d}_{i+1}=\beta_{i} \mathbf{d}_{i}+\mathbf{g}_{\mathrm{cg}, i}
\end{aligned}
$$

End for

Algorithm 1: Basic conjugate gradient algorithm.

properties summarized as follows [12]:

(i) $\mathbf{R}$-orthogonality or conjugacy with respect to $\mathbf{R}$ of the vectors $\mathbf{d}_{i}$, that is, $\mathbf{d}_{i}^{H} \mathbf{R} \mathbf{d}_{j}=0$, for all $i \neq j$,

(ii) the gradient vectors are mutually orthogonal, that is, $\mathbf{g}_{\mathrm{cg}, i}^{H} \mathbf{g}_{\mathrm{cg}, j}=0$, for all $i \neq j$,

(iii) $\mathbf{g}_{\mathrm{cg}, i}^{\mathrm{H}} \mathbf{d}_{j}=0$, for all $j<i$,

(iv) if the gradient vectors $\mathbf{g}_{\mathrm{cg}, i}, i=0, \ldots, D-1$, are normalized, then the transformed covariance matrix $\mathbf{T}_{D}=$ $\mathbf{G}_{\mathrm{cg}, D}^{H} \mathbf{R G}_{\mathrm{cg}, D}$ of dimension $D \times D$ is a real symmetric tridiagonal matrix;

(v) $\mathfrak{D}_{D}=\operatorname{span}\left\{\mathbf{d}_{1}, \mathbf{d}_{2}, \ldots, \mathbf{d}_{D}\right\} \equiv \operatorname{span}\left\{\mathbf{G}_{\mathrm{cg}, D}\right\} \equiv \mathcal{K}^{D}(\mathbf{R}, \mathbf{b})$,

where $\mathcal{K}^{D}(\mathbf{R}, \mathbf{b})=\operatorname{span}\left\{\left[\mathbf{b}, \mathbf{R} \mathbf{b}, \mathbf{R}^{2} \mathbf{b}, \ldots, \mathbf{R}^{D-1} \mathbf{b}\right]\right\}$ is the Krylov subspace of dimension $D$ associated with the pair $(\mathbf{R}, \mathbf{b})[12]$.

After $D$ iterations, the CG algorithm produces an iterative method to solve the reduced rank Wiener solution of (7). Note that the basic idea behind the rank reduction is to project the observation data onto a lower-dimensional subspace $(D<M)$, defined by a set of basis vectors [15]. It is then worth noting that other reduced rank solutions have been obtained via the auxiliary vectors-based (AV) algorithm and the powers of $\mathbf{R}$ (POR) algorithm [15]. These algorithms theoretically and asymptotically yield the same solution as the CG algorithm since they proceed from the same minimization criterion and the same projection subspace [16]. However, as the ways of obtaining the solution differ, these methods are expected to have different performance in practical applications.

In the following, we propose a new DOA estimator from the CG algorithm presented above.

\section{PROPOSED DOA ESTIMATION ALGORITHM}

In this section, the signal model (1)-(5) is considered and an extended signal subspace of rank $P+1$ nonbased on the eigenvector analysis is generated using the same basis proce- dure developed in the work of Grover et al. [13, 14]. Let us define the initial vector $\mathbf{b}(\theta)$ as follows:

$$
\mathbf{b}(\theta)=\frac{\mathbf{R a}(\theta)}{\|\mathbf{R a}(\theta)\|},
$$

where $\mathbf{a}(\theta)$ is a search vector of the form (2) depending on $\theta \in\left[-90^{\circ}, 90^{\circ}\right]$. When the $P$ sources are uncorrelated and $\theta=\theta_{j}$ for $j=1, \ldots, P$, we have

$$
\begin{aligned}
\mathbf{R a}\left(\theta_{j}\right)= & \left(E\left[s_{j}^{2}\right] M+\sigma^{2}\right) \mathbf{a}\left(\theta_{j}\right) \\
& +\sum_{l=1 ; l \neq j}^{P} E\left[s_{l}^{2}\right]\left(\mathbf{a}^{H}\left(\theta_{l}\right) \mathbf{a}\left(\theta_{j}\right)\right) \mathbf{a}\left(\theta_{l}\right) .
\end{aligned}
$$

It appears that $\mathbf{b}\left(\theta_{j}\right)$ is a linear combination of the $P$ signal steering vectors and thus it lies in the signal subspace of dimension $P$. However, when $\theta \neq \theta_{j}$ for $j \in\{1, \ldots, P\}$,

$$
\mathbf{R a}(\theta)=\sum_{j=1}^{P} E\left[s_{j}^{2}\right]\left(\mathbf{a}^{H}\left(\theta_{j}\right) \mathbf{a}(\theta)\right) \mathbf{a}\left(\theta_{j}\right)+\sigma^{2} \mathbf{a}(\theta) .
$$

$\mathbf{b}(\theta)$ is then a linear combination of the $P+1$ steering vectors $\left\{\mathbf{a}(\theta), \mathbf{a}\left(\theta_{1}\right), \mathbf{a}\left(\theta_{2}\right), \ldots, \mathbf{a}\left(\theta_{P}\right)\right\}$ and therefore it belongs to the extended signal subspace of dimension $P+1$ which includes the true signal subspace of dimension $P$ plus the search vector $\mathbf{a}(\theta)$.

For each initial vector described above (10) and after performing $P$ iterations $(D=P)$ of the CG algorithm, we form a set of residual gradient vectors $\left\{\mathbf{g}_{\mathrm{cg}, 0}, \mathbf{g}_{\mathrm{cg}, 1}, \ldots, \mathbf{g}_{\mathrm{cg}, P-1} \mathbf{g}_{\mathrm{cg}, P}\right\}$ (all these vectors are normalized except $\mathbf{g}_{\mathrm{cg}, P}$ ). Therefore, it can be shown (see Appendix A) that if the initial vector $\mathbf{b}(\theta)$ is contained in the signal subspace, then the set of vectors $\mathbf{G}_{\mathrm{cg}, P}=\left\{\mathbf{g}_{\mathrm{cg}, 0}, \mathbf{g}_{\mathrm{cg}, 1}, \ldots, \mathbf{g}_{\mathrm{cg}, P-1}\right\}$ will also be contained in the column space of $\mathbf{A}(\boldsymbol{\Theta})$, hence, the orthonormal matrix $\mathbf{G}_{\mathrm{cg}, P}{ }^{1}$ spans the true signal subspace for $\theta=\theta_{j}, j=1,2, \ldots, P$, that is,

$$
\operatorname{span}\left\{\mathbf{G}_{\mathrm{cg}, P}\right\} \equiv \operatorname{span}\{\mathbf{A}(\boldsymbol{\Theta})\}
$$

and the solution vector $\mathbf{w}=\mathbf{R}^{-1} \mathbf{b}=\mathbf{a}(\theta) /\|\mathbf{R a}(\theta)\|$ also lies in the signal subspace

$$
\mathbf{w} \in \operatorname{span}\left\{\mathbf{g}_{\mathrm{cg}, 0}, \mathbf{g}_{\mathrm{cg}, 1}, \ldots, \mathbf{g}_{\mathrm{cg}, P-1}\right\}
$$

\footnotetext{
${ }^{1}$ If we perform an eigendecomposition of the tridiagonal matrix $\mathbf{T}_{P}=$ $\mathbf{G}_{\mathrm{cg}, P}^{H} \mathbf{R G}_{\mathrm{cg}, P}$, we have $\mathbf{T}_{P}=\sum_{i=1}^{P} \lambda_{i} \mathbf{e}_{i} \mathbf{e}_{i}^{H}$, then the $P$ eigenvalues $\lambda_{i}, i=$ $1, \ldots, P$, of $\mathbf{T}_{P}$ are the $P$ principal eigenvalues of the covariance matrix $\mathbf{R}$, and the vectors $\mathbf{y}_{i}=\mathbf{G}_{\mathrm{cg}, P} \mathbf{e}_{i}, i=1, \ldots, P$, (where $\mathbf{e}_{i}$ is the $i$ th eigenvector of $\mathbf{T}_{P}$ and $\mathbf{y}_{i}$ are the Rayleigh-Ritz vectors associated with $\mathcal{K}^{D}(\mathbf{R}, \mathbf{b})$ ) are asymptotically equivalent to the principal eigenvectors of $\mathbf{R}$ [17].
} 
Now, when $\theta \neq \theta_{j}$ for $j \in\{1, \ldots, P\}, \mathbf{G}_{\mathrm{cg}, P+1}{ }^{2}$ spans the extended subspace yielding (see Appendix A)

$$
\operatorname{span}\left\{\mathbf{G}_{\mathrm{cg}, P+1}\right\} \equiv \operatorname{span}\{\mathbf{A}(\boldsymbol{\Theta}), \mathbf{a}(\theta)\} .
$$

In this case, $\mathbf{w}$ is also in the extended signal subspace, that is,

$$
\mathbf{w} \in \operatorname{span}\left\{\mathbf{g}_{\mathrm{cg}, 0}, \mathbf{g}_{\mathrm{cg}, 1}, \ldots, \mathbf{g}_{\mathrm{cg}, P}\right\}
$$

Proposition 1. After $P$ iterations of the CG algorithm the following equality holds for $\theta=\theta_{j}, j=1,2, \ldots, P$ :

$$
\mathbf{g}_{c g, P}^{H}(\theta)=0,
$$

where $\mathbf{g}_{c, P, P}$ is the residual CG vector left unnormalized at iteration $P$.

Proof. Since the gradient vectors $\mathbf{g}_{\mathrm{cg}, i}$ generated by the CG algorithm are orthogonal [12], $\operatorname{span}\left\{\mathbf{g}_{\mathrm{cg}, 0}, \mathbf{g}_{\mathrm{cg}, 1}, \ldots, \mathbf{g}_{\mathrm{cg}, P}\right\}$ is of rank $P+1$. Using the fact that when $\theta=\theta_{j}, j=1,2, \ldots, P$,

$$
\operatorname{span}\left\{\mathbf{g}_{\mathrm{cg}, 0}, \mathbf{g}_{\mathrm{cg}, 1}, \ldots, \mathbf{g}_{\mathrm{cg}, P-1}\right\}=\operatorname{span}\{\mathbf{A}(\boldsymbol{\Theta})\}
$$

Then

$$
\operatorname{span}\left\{\mathbf{g}_{\mathrm{cg}, 0}, \mathbf{g}_{\mathrm{cg}, 1}, \ldots, \mathbf{g}_{\mathrm{cg}, P-1}, \mathbf{g}_{\mathrm{cg}, P}\right\}=\operatorname{span}\left\{\mathbf{A}(\boldsymbol{\Theta}), \mathbf{g}_{\mathrm{cg}, P}\right\}
$$

From Appendix A, it is shown that each residual gradient vector generated by the $C G$ algorithm when the initial vector is in the signal subspace $\operatorname{span}\{\mathbf{A}(\Theta)\}$ will also belong to the signal subspace. This is then the case for $\mathbf{g}_{\mathrm{cg}, P}$. Therefore, the rank of $\operatorname{span}\left\{\mathbf{g}_{\mathrm{cg}, 0}, \mathbf{g}_{\mathrm{cg}, 1}, \ldots, \mathbf{g}_{\mathrm{cg}, P-1}, \mathbf{g}_{\mathrm{cg}, P}\right\}$ reduces to $P$ yielding that in this case $\mathbf{g}_{\mathrm{cg}, P}$ should be zero or a linear combination of the other gradient vectors which is not possible since it is orthogonal to all of them.

In view of Proposition 1, we use the following localization function as defined in [14, equation (22)]:

$$
\mathcal{P}_{K}\left(\theta^{(n)}\right)=\frac{1}{\left\|\mathbf{g}_{\mathrm{cg}, P}^{H}\left(\theta^{(n)}\right) \mathbf{G}_{\mathrm{cg}, P+1}\left(\theta^{(n-1)}\right)\right\|^{2}},
$$

where $\mathbf{G}_{\mathrm{cg}, P+1}\left(\theta^{(n)}\right)$ is the matrix calculated at step $n$ by performing $D=P$ iterations of the CG algorithm with initial

\footnotetext{
${ }^{2}$ We can show that the eigenvalues of the $(P+1) \times(P+1)$ matrix $\mathbf{T}_{P+1}=$ $\mathbf{G}_{\mathrm{cg}, P+1}^{H} \mathbf{R G}_{\mathrm{cg}, P+1}$ (the last vector $\mathbf{g}_{\mathrm{cg}, P}$ is normalized) are $\left\{\lambda_{1}, \ldots, \lambda_{P}, \sigma^{2}\right\}$, where the eigenvalues $\lambda_{i}, i=1, \ldots, P$, are the $P$ principal eigenvalues of $\mathbf{R}$ and $\sigma^{2}$ is the smallest eigenvalue of $\mathbf{R}$. The first $P$ RR vectors from the set $\mathbf{y}_{i}=\mathbf{G}_{\mathrm{cg}, P+1} \mathbf{e}_{i}, i=1, \ldots, P$, are asymptotically equivalents to the principal eigenvectors of $\mathbf{R}$ [17], and the last (RR) vector associated to $\sigma^{2}$ is orthogonal to the principal eigenspace (belonging to the noise subspace), that is, $\mathbf{y}_{P+1}^{H} \mathbf{A}(\theta)=\mathbf{0}$.
}

residual vector $\mathbf{g}_{\mathrm{cg}, 0}\left(\theta^{(n)}\right)=\mathbf{b}\left(\theta^{(n)}\right)$ as defined in (10), that is,

$$
\mathbf{G}_{\mathrm{cg}, P+1}\left(\theta^{(n)}\right)=\left[\mathbf{g}_{\mathrm{cg}, 0}\left(\theta^{(n)}\right), \mathbf{g}_{\mathrm{cg}, 1}\left(\theta^{(n)}\right), \ldots, \mathbf{g}_{\mathrm{cg}, P}\left(\theta^{(n)}\right)\right]
$$

$\theta^{(n)}=n \Delta$ with $n=1,2,3, \ldots, 180^{\circ} / \Delta^{\circ}$ and $\Delta$ is the search angle step.

Note that the choice of using $1 /\left\|\mathbf{g}_{\mathrm{cg}, P}\left(\theta^{(n)}\right)\right\|^{2}$ as a localization function was first considered. Since the results were not satisfactory enough, (20) was finally preferred. According to the modified orthonormal AV [16], the normalized gradient $\mathrm{CG}$ and the $\mathrm{AV}$ are identical because the AV recurrence is formally the same as Lanczos recurrence [12]. Thus, if the initial vector $\mathbf{g}_{\mathrm{cg}, 0}$ in CG algorithm is parallel to the initial vector in $\mathrm{AV}$, then all successive normalized gradients in CG will be parallel to the corresponding AV vectors (see Appendix B). Let $\mathbf{g}_{\mathrm{av}, i}, i=0, \ldots, P-1$, represent the orthonormal basis in $\mathrm{AV}$ procedure and the last unormalized vectors by $\mathbf{g}_{\mathrm{av}, P}$. Then, it is easy to show that the CG spectra are related to the AV spectra by

$$
\begin{aligned}
& \mathcal{P}_{K}\left(\theta^{(n)}\right)=\left(\left|c_{P}\left(\theta^{(n)}\right)\right|^{2}\right. \\
& \times\left(\left\|\mathbf{g}_{\mathrm{av}, p}^{H}\left(\theta^{(n)}\right) \mathbf{g}_{\mathrm{av}, 0}\left(\theta^{(n-1)}\right)\right\|^{2}\right. \\
& \quad+\cdots+\left|c_{P}\left(\theta^{(n-1)}\right)\right|^{2} \\
&\left.\left.\quad \times\left\|\mathbf{g}_{\mathrm{av}, p}^{H}\left(\theta^{(n)}\right) \mathbf{g}_{\mathrm{av}, P}\left(\theta^{(n-1)}\right)\right\|^{2}\right)\right)^{-1},
\end{aligned}
$$

where

$$
\left|c_{P}\left(\theta^{(n)}\right)\right|=\frac{\| \mathbf{g}_{\mathrm{cg}, P}\left(\theta^{(n)}\right)||}{\left|\mu_{P-1}\left(\theta^{(n)}\right)\right|} \frac{\alpha_{P}\left(\theta^{(n)}\right)}{\sqrt{\beta_{P}\left(\theta^{(n)}\right)}},
$$

the difference, therefore, between the AV $[13,14]$ and CG spectra is the scalars $\left|c_{P}\left(\theta^{(n)}\right)\right|^{2}$ calculated at steps $n-1$ and $n$ due to the last basis vector that is unnormalized (see Appendix B for the details). It is easy to show that we can obtain a peak in the spectrum if $\theta^{(n)}=\theta_{j}, j=1, \ldots, P$, because the last vector in the basis $\mathbf{g}_{\mathrm{cg}, P}\left(\theta^{(n)}\right)=0$. However, when $\theta^{(n)} \neq \theta_{j}, j=1, \ldots, P, \mathbf{g}_{\mathrm{cg}, P}\left(\theta^{(n)}\right)$ is contained in the extended signal subspace $\operatorname{span}\left\{\mathbf{A}(\boldsymbol{\Theta}), \mathbf{a}\left(\theta^{(n)}\right)\right\}$ and the following relation holds:

$$
\operatorname{span}\left\{\mathbf{G}_{\mathrm{cg}, P+1}\left(\theta^{(n-1)}\right)\right\}=\operatorname{span}\left\{\mathbf{A}(\Theta), \mathbf{a}\left(\theta^{(n-1)}\right)\right\} .
$$

We can note that $\left\|\mathbf{g}_{\mathrm{c}, P}^{H}\left(\theta^{(n)}\right) \mathbf{G}_{\mathrm{cg}, P+1}\left(\theta^{(n-1)}\right)\right\| \neq 0$ except when $\mathbf{g}_{\mathrm{cg}, P}\left(\theta^{(n)}\right)$ is proportional to $\mathbf{a}\left(\theta^{(n)}\right)$ and $\mathbf{a}\left(\theta^{(n)}\right)$ is orthogonal both to $\mathbf{A}(\Theta)$ and $\mathbf{a}\left(\theta^{(n-1)}\right)$ which can be considered as a very rare situation in most cases.

In real situations, $\mathbf{R}$ is unknown and we use rather the sample average estimate $\widehat{\mathbf{R}}$ as defined in (6). From (20), it is clear that when $\theta^{(n)}=\theta_{j}, j=1, \ldots, P$, we will have $\left\|\hat{\mathbf{g}}_{\mathrm{cg}, \mathrm{P}}^{H}\left(\theta^{(n)}\right) \hat{\mathbf{G}}_{\mathrm{cg}, P+1}\left(\theta^{(n-1)}\right)\right\|$ not equal to zero but very small and $\widehat{\mathcal{P}}_{K}\left(\theta^{(n)}\right)$ very large but not infinite.

Concerning the computational complexity, it is worth noting that the proposed algorithm (it is also the case for the AV-based algorithm proposed in $[13,14])$ is more complex 
than MUSIC since the gradient vectors forming the signal subspace basis necessary to construct the pseudospectrum must be calculated for each search angle. The proposed algorithm is therefore interesting for applications where a very high resolution capability is required in the case of a small number of snapshots and a low signal-to-noise ratio (SNR). This will be demonstrated through intensive simulations in the next section. Also note that when the search angle area is limited, the new algorithm has a comparable computational complexity as MUSIC.

\section{SIMULATION RESULTS}

In this section, computer simulations were conducted with a uniform linear array composed of 10 isotropic sensors whose spacing equals half-wavelength. There are two equal-power plane waves arriving on the array. The internal noises of equal power exist at each sensor element and they are statistically independent of the incident signal and of each other. Angles of arrival are measured from the broadside direction of the array. First, we fix the signal angles of arrival at $-1^{\circ}$ and $1^{\circ}$ and the SNR's at $10 \mathrm{~dB}$. In Figure 1, we examine the proposed localization function or pseudo-spectrum when the observation data record $K=50$ compared with that of the AV-based algorithm [13, 14, 18, 19] and of MUSIC. The CG pseudo-spectrum resolves the two sources better than the AV algorithm where the MUSIC algorithm completely fails. Notice that the higher gain of CG method is due to the factor $c_{p}$ which depends on the norm of the gradient.

In the following, in order to analyze the performance of the algorithms in terms of the resolution probability, we use the following random inequality [20]:

$$
\mathcal{P}_{K}\left(\theta_{m}\right)-\frac{1}{2}\left(\mathcal{P}_{K}\left(\theta_{1}\right)+\mathcal{P}_{K}\left(\theta_{2}\right)\right)<0,
$$

where $\theta_{1}$ and $\theta_{2}$ are the angles of arrivals of the two signals and $\theta_{m}$ denotes their mean. $\mathcal{P}_{K}(\theta)$ is the pseudo-spectrum defined in (20) as a function of the angle of arrival $\theta$.

To illustrate the performance of the proposed algorithm two experiments were conducted.

Experiment 1 (uncorrelated sources). In this experiment, we consider the presence of two uncorrelated complex Gaussian sources separated by $3^{\circ}$. In Figures 2 and 3, we show the probability of resolution of the algorithms as a function of the SNR (when $K=50$ ) and the number of snapshots (with SNR $=0 \mathrm{~dB}$ ), respectively. For purpose of comparisons, we added the ESPRIT algorithm [3]. As expected, the resolution capability of all the algorithms increases as we increase the number of snapshots $K$ and the SNR. We also clearly note the complete failure of MUSIC as well as ESPRIT to resolve the two signals compared to the two algorithms CG and AV (Krylov subspace-based algorithms). The two figures show that the CG-based algorithms outperforms its counterparts in terms of resolution probability.

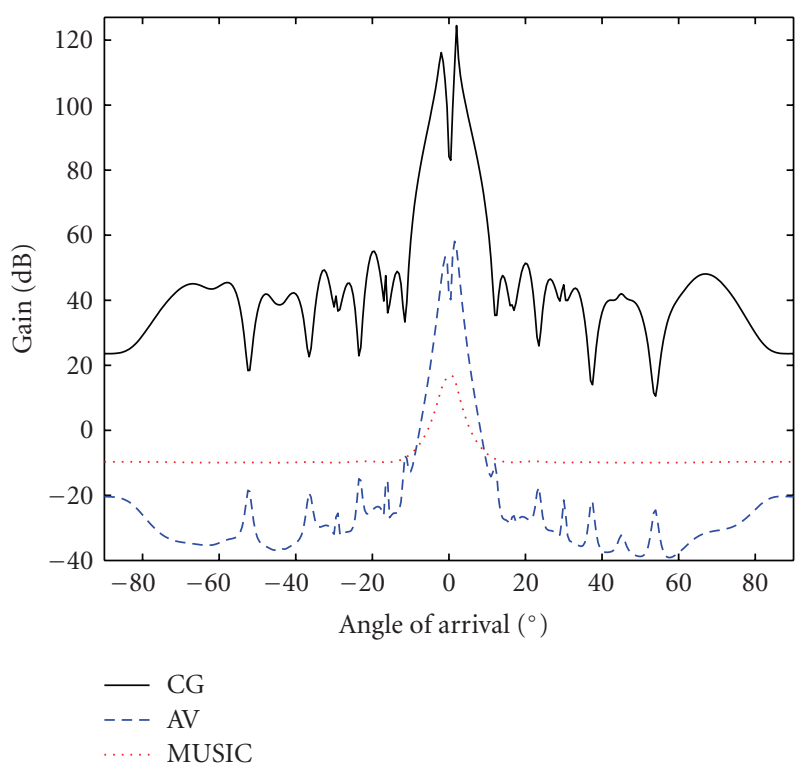

Figure 1: CG, AV, and MUSIC spectra $\left(\theta_{1}=-1^{\circ}, \theta_{2}=1^{\circ}, \mathrm{SNR} 1=\right.$ $\mathrm{SNR} 2=10 \mathrm{~dB}, K=50$ ).

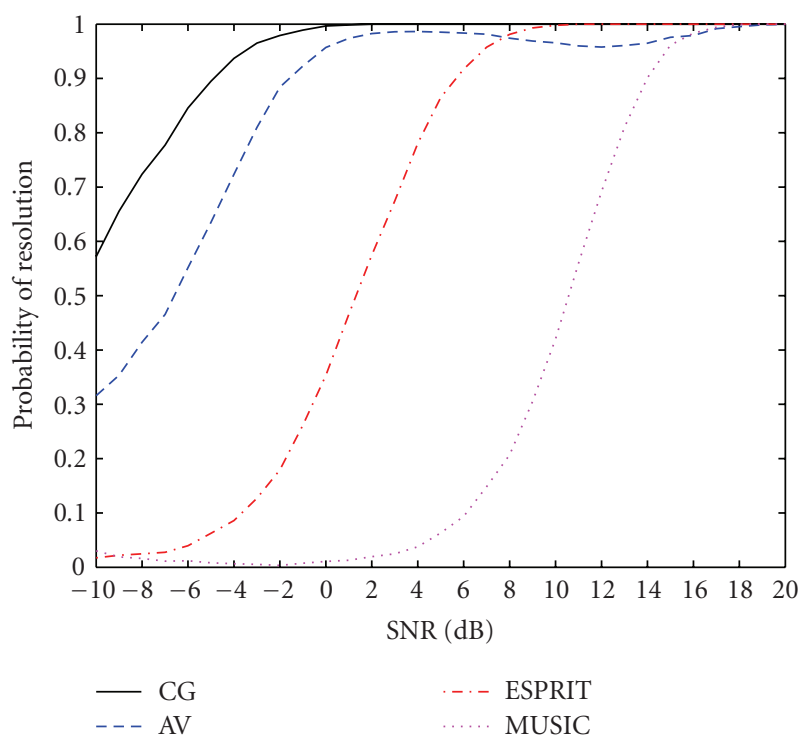

FIGURE 2: Probability of resolution versus SNR (separation $3^{\circ}, K=$ $50)$.

Experiment 2 (correlated sources). In this experiment, we consider the presence of two correlated random complex Gaussian sources generated as follows:

$$
\mathbf{s}_{1} \sim \mathcal{N}\left(0, \sigma_{S}^{2}\right), \quad \mathbf{s}_{2}=r \mathbf{s}_{1}+\sqrt{1-r^{2}} \mathbf{s}_{3}
$$

where $\boldsymbol{s}_{3} \sim \mathcal{N}\left(0, \sigma_{S}^{2}\right)$ and $r$ is the correlation coefficient. Figures 4 and 5 show the probability of resolution of the algorithms for high correlation value $r=0.7$ with and without 


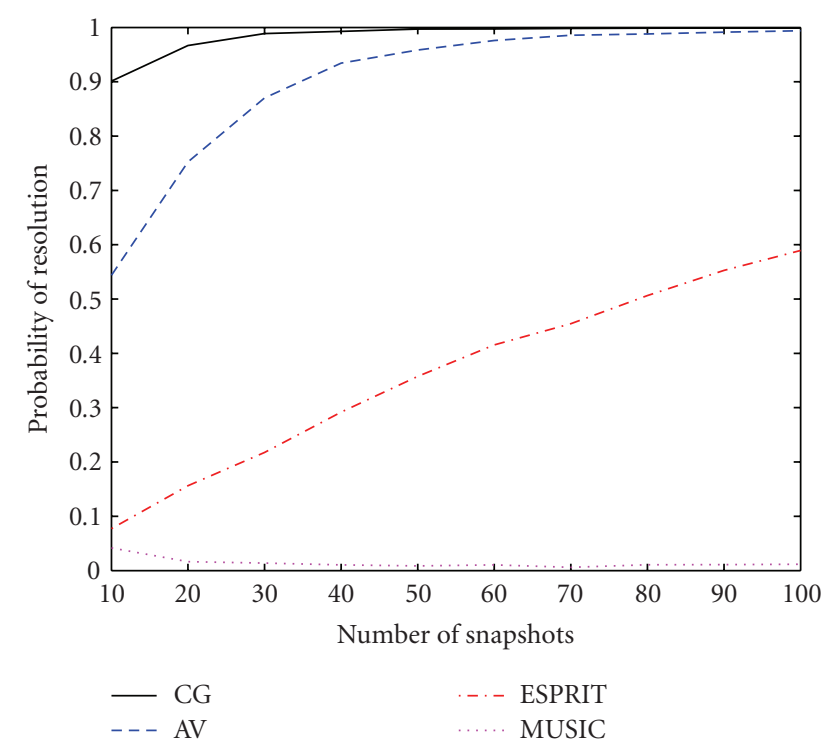

FIgURe 3: Probability of resolution versus number of snapshots (separation $3^{\circ}, \mathrm{SNR}=0 \mathrm{~dB}$ ).

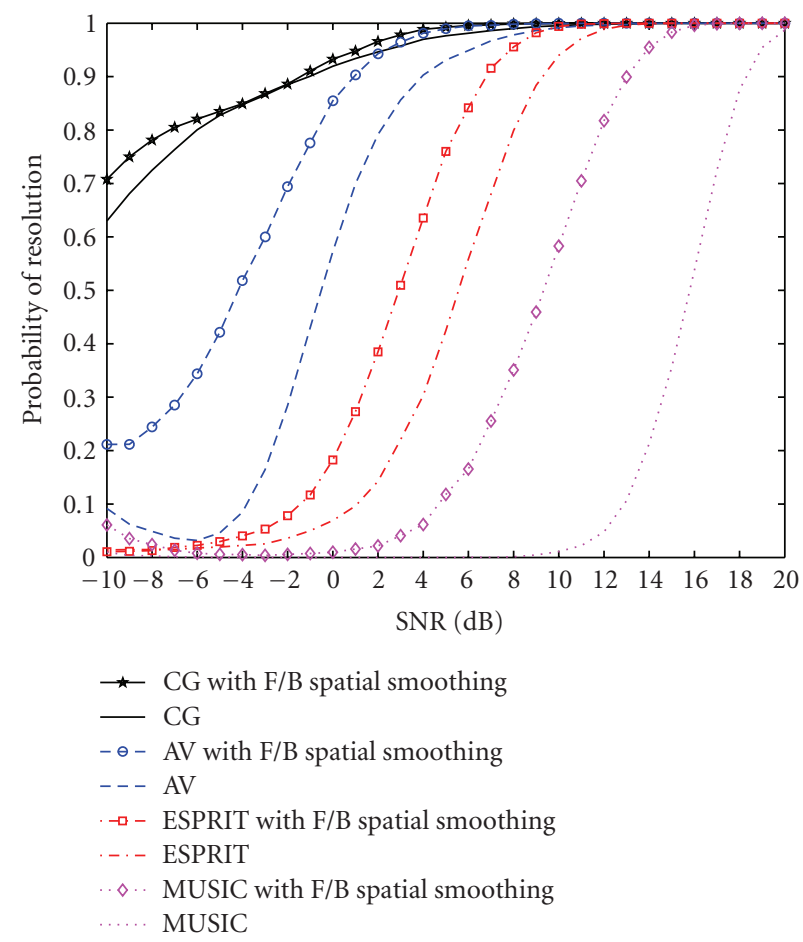

Figure 4: Probability of resolution versus SNR (separation $3^{\circ}, K=$ $50, r=0.7)$.

forward/backward spatial smoothing (FBSS) [21]. Figure 4 plots the probability of resolution versus SNR for a fixed record data $K=50$ and Figure 5 plots the probability of resolution versus number of snapshots for an $\mathrm{SNR}=5 \mathrm{~dB}$. The two figures demonstrate that the CG-basis estimator still outperforms the AV-basis estimator in probability of resolu-

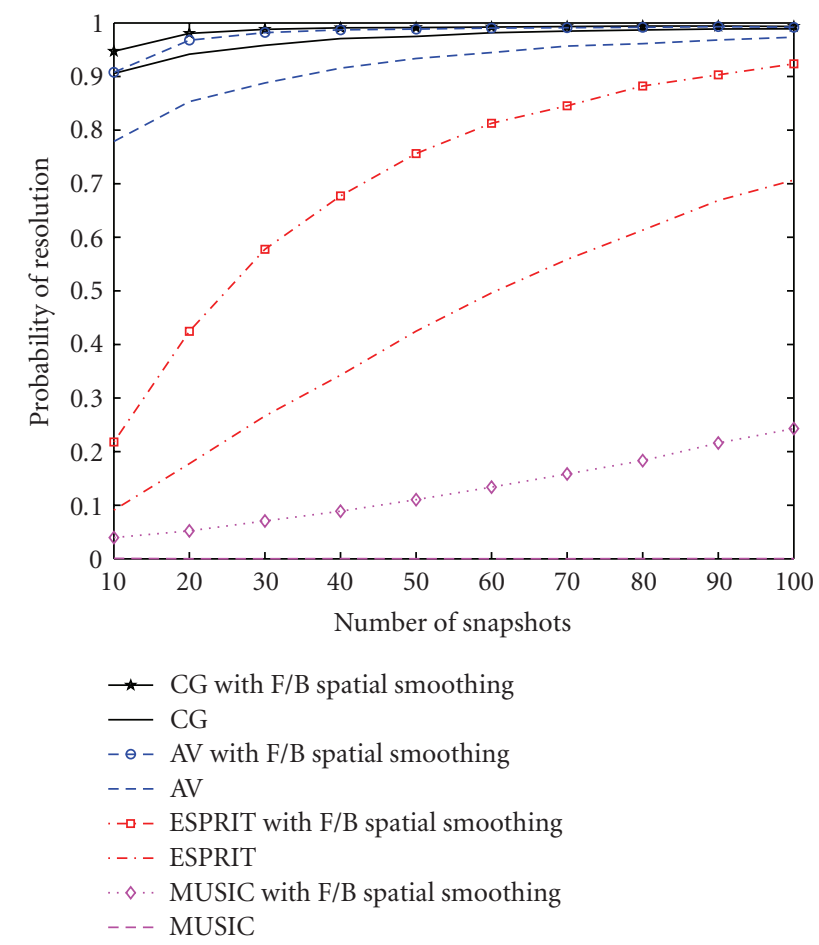

Figure 5: Probability of resolution versus number of snapshots (separation $3^{\circ}, \mathrm{SNR}=5 \mathrm{~dB}, r=0.7$ ).

tion in the case of correlated sources with or without FBSS. We also note that the CG-based and the AV-based estimators (without FBSS) have better performance than MUSIC and ESPRIT with FBSS, at low SNR and whatever the record data size (Figure 5).

Finally, we repeat the previous simulations for highly correlated sources $(r=0.9)$. At low SNR (see Figure 6), we show that the CG-based method even without FBSS still achieves better results than the AV-based method and over MUSIC and ESPRIT with or without FBSS $(<8 \mathrm{~dB}$ for ESPRIT with spatial smoothing). In Figure 7, the proposed algorithm reveals again higher performance over MUSIC and ESPRIT with or without FBSS; which is unlike its counterpart the AV-based algorithm where it has less resolution capability compared to ESPRTI with FBSS for data record $K<70$. We can also notice the improvement of resolution probability for both the CG and AV-based algorithms with FBSS.

\section{CONCLUSION}

In this paper, the application of the CG algorithm to the DOA estimation problem has been proposed. The new method does not resort to the eigendecomposition of the observation data covariance matrix. Instead, it uses a new basis for the signal subspace based on the residual vectors of the CG algorithm. Numerical results indicate that the proposed algorithm outperforms its counterparts which are the $\mathrm{AV}$ 


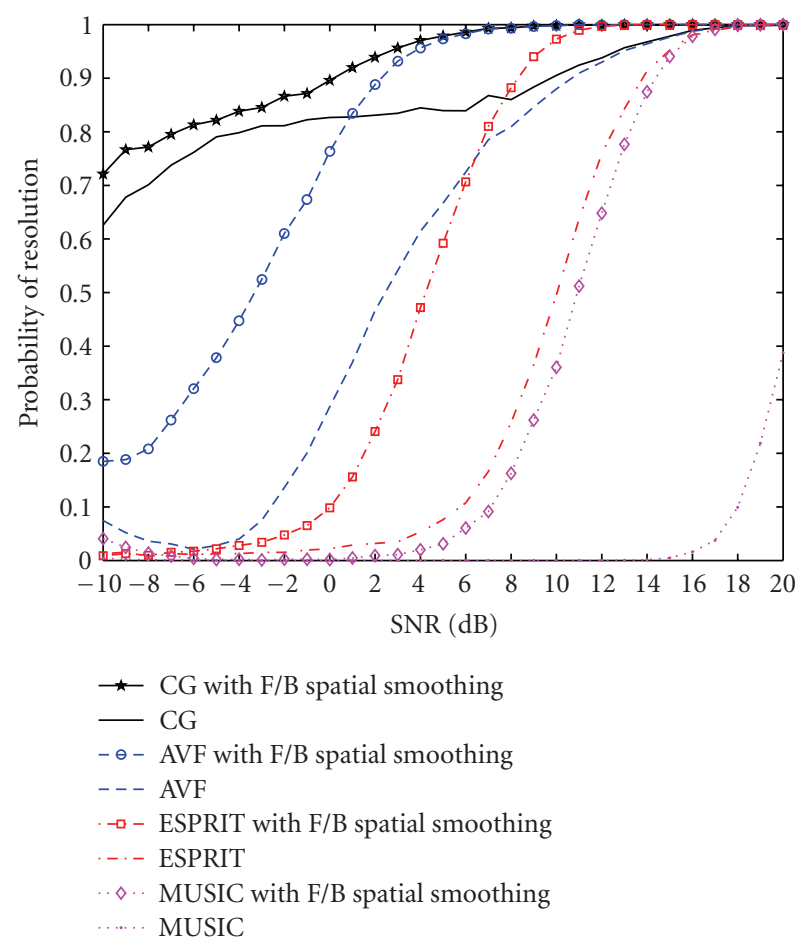

FIgURe 6: Probability of resolution versus SNR (separation $3^{\circ}, K=$ $50, r=0.9)$.

algorithm, the classical MUSIC and ESPRIT, in terms of resolution capacity at a small record data and low SNR.

\section{APPENDICES}

\section{A.}

Let us assume that $\mathbf{b}(\theta) \in \operatorname{span}\{\mathbf{A}(\boldsymbol{\Theta}), \mathbf{a}(\theta)\}$. It follows from Algorithm 1 that

$$
\mathbf{g}_{\mathrm{cg}, 1}=\mathbf{b}(\theta)-\alpha_{1} \mathbf{R b}(\theta)
$$

also belongs to $\operatorname{span}\{\mathbf{A}(\boldsymbol{\Theta}), \mathbf{a}(\theta)\}$ since

$$
\mathbf{R b}(\theta)=\sum_{j=1}^{P} E\left[s_{j}^{2}\right]\left(\mathbf{a}\left(\theta_{j}\right)^{H} \mathbf{b}(\theta)\right) \mathbf{a}\left(\theta_{j}\right)+\sigma^{2} \mathbf{b}(\theta)
$$

is a linear combination of vectors of $\operatorname{span}\{\mathbf{A}(\boldsymbol{\Theta}), \mathbf{a}(\theta)\}$. Then $\mathbf{d}_{2}=\mathbf{g}_{\mathrm{cg}, 1}-\beta_{1} \mathbf{d}_{1}$ also belongs to $\operatorname{span}\{\mathbf{A}(\boldsymbol{\Theta}), \mathbf{a}(\theta)\}$ (with $\mathbf{d}_{1}=$ $\mathbf{b}(\theta))$. In the same way, we have

$$
\mathbf{g}_{\mathrm{cg}, 2}=\mathbf{g}_{\mathrm{cg}, 1}-\alpha_{2} \mathbf{v}_{2}
$$

with

$$
\mathbf{v}_{2}=\mathbf{R d}_{2}
$$

also belonging to the extended signal subspace since

$$
\mathbf{R d}_{2}=\sum_{j=1}^{P} E\left[s_{j}^{2}\right]\left(\mathbf{a}\left(\theta_{j}\right)^{H} \mathbf{d}_{2}(\theta)\right) \mathbf{a}\left(\theta_{j}\right)+\sigma^{2} \mathbf{d}_{2}(\theta) .
$$

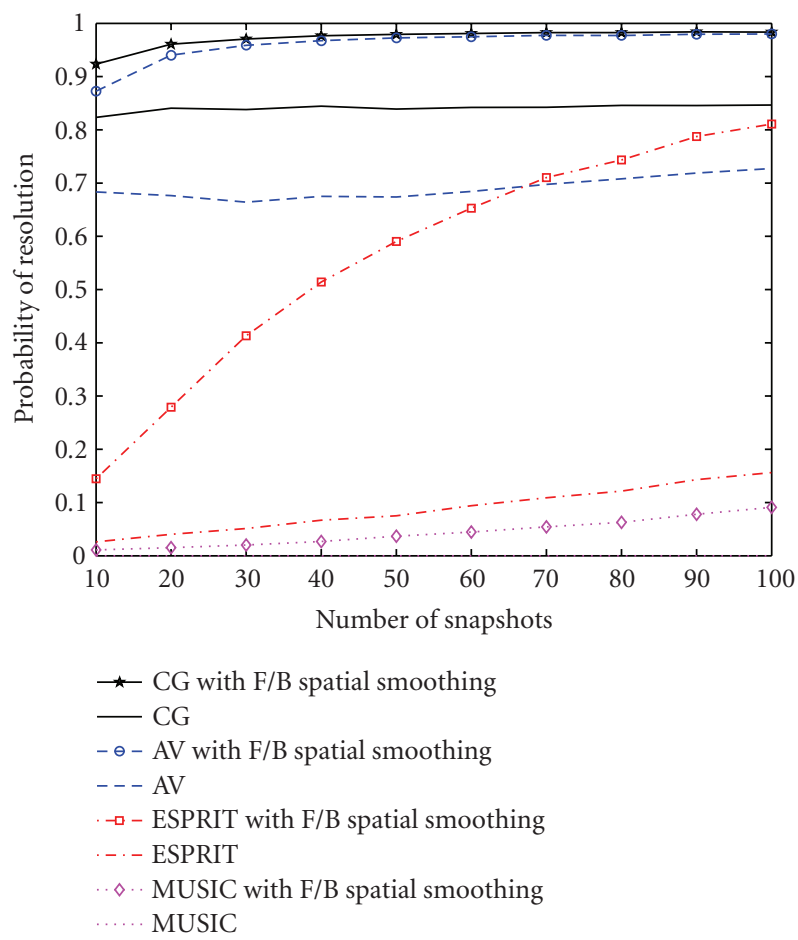

FIgURe 7: Probability of resolution versus number of snapshots (separation $3^{\circ}, \mathrm{SNR}=5 \mathrm{~dB}, r=0.9$ ).

More generally, it is then easy to check that when $\mathbf{g}_{\mathrm{cg}, i-1}$ and $\mathbf{d}_{\mathrm{cg}, i-1}$ are vectors of $\operatorname{span}\{\mathbf{A}(\boldsymbol{\Theta}), \mathbf{a}(\theta)\}$, then $\mathbf{g}_{\mathrm{cg}, i}$ and $\mathbf{d}_{\mathrm{cg}, i}$ are also vectors of $\operatorname{span}\{\mathbf{A}(\boldsymbol{\Theta}), \mathbf{a}(\theta)\}$. Now when $\theta=\theta_{j}$, the extended subspace reduces to $\operatorname{span}\{\mathbf{A}(\boldsymbol{\Theta})\}$.

B.

Let $\mathbf{g}_{\mathrm{av}, i}$ be the auxiliary vector (AV) $[18,19]$; it was shown in [16] that a simplified recurrence for $\mathbf{g}_{\mathrm{av}, i+1}, i \geq 1$, is given by

$$
\begin{aligned}
& \mathbf{g}_{\mathrm{av}, i+1}=\frac{\left(\mathbf{I}-\sum_{l=i-1}^{i} \mathbf{g}_{\mathrm{av}, l} \mathbf{g}_{\mathrm{av}, l}^{\mathrm{H}}\right) \mathbf{R g}_{\mathrm{av}, i}}{\left\|\left(\mathbf{I}-\sum_{l=i-1}^{i} \mathbf{g}_{\mathrm{av}, l} \mathbf{g}_{\mathrm{av}, l}^{H}\right) \mathbf{R g}_{\mathrm{av}, i}\right\|}, \\
& \mathbf{g}_{\mathrm{av}, 1}=\frac{\mathbf{R g}_{\mathrm{av}, 0}-\mathbf{g}_{\mathrm{av}, 0}\left(\mathbf{g}_{\mathrm{av}, 0}^{\mathrm{H}} \mathbf{R g}_{\mathrm{av}, 0}\right)}{\left\|\mathbf{R g}_{\mathrm{av}, 0}-\mathbf{g}_{\mathrm{av}, 0}\left(\mathbf{g}_{\mathrm{av}, 0}^{\mathrm{H}} \mathbf{R g}_{\mathrm{av}, 0}\right)\right\|},
\end{aligned}
$$

where $\mathbf{g}_{\mathrm{av}, 0}$ is the first vector in the AV basis. Notice that the auxiliary vectors are restricted to be orthonormal in contrast to the nonorthogonal AV work in $[19,22]$. Recall that if initial vectors are equals, that is,

$$
\mathbf{g}_{\mathrm{av}, 0}=\mathbf{g}_{\mathrm{cg}, 0}=\mathbf{b}(\theta),
$$

then it is easy to show from Algorithm 1 that

$$
\frac{\mathbf{g}_{\mathrm{cg}, 1}}{\left\|\mathbf{g}_{\mathrm{cg}, 1}\right\|}=-\frac{\mathbf{R g}_{\mathrm{cg}, 0}-\mathbf{g}_{\mathrm{cg}, 0}\left(\mathbf{g}_{\mathrm{cg}, 0}^{\mathrm{H}} \mathbf{R} \mathbf{g}_{\mathrm{cg}, 0}\right)}{\left\|\mathbf{R g}_{\mathrm{cg}, 0}-\mathbf{g}_{\mathrm{cg}, 0}\left(\mathbf{g}_{\mathrm{cg}, 0}^{H} \mathbf{R g}_{\mathrm{cg}, 0}\right)\right\|}=-\mathbf{g}_{\mathrm{av}, 1} .
$$


From (B.1) we can obtain

$$
\begin{aligned}
\left\|\mathbf{t}_{i}\right\| \mathbf{g}_{\mathrm{av}, i+1} & =\mathbf{R g}_{\mathrm{av}, i}-\left(\mathbf{g}_{\mathrm{av}, i}^{H} \mathbf{R g}_{\mathrm{av}, i}\right) \mathbf{g}_{\mathrm{av}, i}-\left(\mathbf{g}_{\mathrm{av}, i-1}^{H} \mathbf{R g}_{\mathrm{av}, i}\right) \mathbf{g}_{\mathrm{av}, i-1} \\
\delta_{i} \mathbf{g}_{\mathrm{av}, i+1} & =\mathbf{R g}_{\mathrm{av}, i}-\gamma_{i} \mathbf{g}_{\mathrm{av}, i}-\delta_{i-1} \mathbf{g}_{\mathrm{av}, i-1} .
\end{aligned}
$$

Thus, the last equation (B.5) is the well-known Lanczos recurrence [12], where $\left\|\mathbf{t}_{i}\right\|=\left\|\left(\mathbf{I}-\sum_{l=i-1}^{i} \mathbf{g}_{\mathrm{av}, l} \mathbf{g}_{\mathrm{av}, l}^{H}\right) \mathbf{R} \mathbf{g}_{\mathrm{av}, i}\right\|$ and the coefficients $\gamma_{i}$ and $\delta_{i}$ are the elements of the tridiagonal matrix $\mathbf{G}_{\mathrm{av}, i}^{H} \mathbf{R G}_{\mathrm{av}, i}$, where $\mathbf{G}_{\mathrm{av}, i}$ is the matrix formed by the $i$ normal AV vectors. From the interpretation of Lanczos algorithm, if the initial gradient CG algorithm $\mathbf{g}_{\mathrm{cg}, 0}$ is parallel to the initial $\mathbf{g}_{\mathrm{av}, 0}$, then all successive normalized gradients in CG are the same as the AV algorithm [12], that is,

$$
\mathbf{g}_{\mathrm{av}, i}=(-1)^{i} \frac{\mathbf{g}_{\mathrm{cg}, i}}{\left\|\mathbf{g}_{\mathrm{cg}, i}\right\|}, \quad i \geq 1 .
$$

From the expression for the CG algorithm, we can express the gradient vectors $\mathbf{g}_{\mathrm{cg}, i+1}$ in terms of the previous gradient vectors using line 6 and 9 of Algorithm 1, then we can write

$$
\frac{\mathbf{g}_{\mathrm{cg}, i+1}}{\alpha_{i+1}}=-\mathbf{R} \mathbf{g}_{\mathrm{cg}, i}+\left(\frac{1}{\alpha_{i+1}}+\frac{\beta_{i}}{\alpha_{i}}\right) \mathbf{g}_{\mathrm{cg}, i}-\frac{\beta_{i}}{\alpha_{i}} \mathbf{g}_{\mathrm{cg}, i-1} .
$$

Multiplying and dividing each term of (B.7) by the norm of the corresponding gradient vector results in [23]

$$
\begin{aligned}
& \frac{\sqrt{\beta_{i+1}}}{\alpha_{i+1}} \frac{\mathbf{g}_{\mathrm{cg}, i+1}}{\left\|\mathbf{g}_{\mathrm{cg}, i+1}\right\|} \\
& \quad=-\mathbf{R} \frac{\mathbf{g}_{\mathrm{cg}, i}}{\left\|\mathbf{g}_{\mathrm{cg}, i}\right\|}+\left(\frac{1}{\alpha_{i+1}}+\frac{\beta_{i}}{\alpha_{i}}\right) \frac{\mathbf{g}_{\mathrm{cg}, i}}{\left\|\mathbf{g}_{\mathrm{cg}, i}\right\|}-\frac{\sqrt{\beta_{i}}}{\alpha_{i}} \frac{\mathbf{g}_{\mathrm{cg}, i-1}}{\left\|\mathbf{g}_{\mathrm{cg}, i-1}\right\|} .
\end{aligned}
$$

If (B.8) is identified with (B.5), it yields

$$
\begin{aligned}
\delta_{i} & =\frac{\sqrt{\beta_{i+1}}}{\alpha_{i+1}}, \\
\gamma_{i} & =\frac{1}{\alpha_{i+1}}+\frac{\beta_{i}}{\alpha_{i}}, \quad i \geq 1, \\
\gamma_{1} & =\mathbf{g}_{\mathrm{av}, 0}^{H} \operatorname{Rg}_{\mathrm{av}, 0}=\frac{1}{\alpha_{1}} .
\end{aligned}
$$

We will now prove the relation between the unormalized last vectors $\mathbf{g}_{\mathrm{cg}, P}$ and $\mathbf{g}_{\mathrm{av}, P}$. From [13], the last unnormalized vector in $\mathrm{AV}$ algorithm is given by

$$
\mathbf{g}_{\mathrm{av}, P}=(-1)^{P+1} \mu_{P-1}\left(\mathbf{I}-\sum_{l=P-1}^{P-2} \mathbf{g}_{\mathrm{av}, l} \mathbf{g}_{\mathrm{av}, l}^{H}\right) \mathbf{R g}_{\mathrm{av}, P-1},
$$

where

$$
\begin{gathered}
\mu_{i}=\mu_{i-1} \frac{\mathbf{g}_{\mathrm{av}, i}^{H} \mathbf{R g}_{\mathrm{av}, i-1}}{\mathbf{g}_{\mathrm{av}, i}^{H} \mathbf{R g}_{\mathrm{av}, i}}, \quad i>1, \\
\mu_{1}=\frac{\mathbf{g}_{\mathrm{av}, 1}^{H} \mathbf{R g}_{\mathrm{av}, 0}}{\mathbf{g}_{\mathrm{av}, 1}^{H} \mathbf{R g}_{\mathrm{av}, 1}} .
\end{gathered}
$$

Using (B.5) and (B.9), (B.12) can be rewritten as

$$
\mu_{1}=\frac{\delta_{1}}{\gamma_{2}}=\frac{\sqrt{\beta_{2}}}{\alpha_{2}}\left(\frac{1}{\alpha_{2}}+\frac{\beta_{1}}{\alpha_{1}}\right)^{-1}
$$

and a new recurrence for $\mu_{i}$ can be done with the CG coefficients as

$$
\mu_{i}=\mu_{i-1} \frac{\sqrt{\beta_{i+1}}}{\alpha_{i+1}}\left(\frac{1}{\alpha_{i+1}}+\frac{\beta_{i}}{\alpha_{i}}\right)^{-1}, \quad i>1,
$$

hence from (B.6), we can obtain

$$
\mathbf{g}_{\mathrm{cg}, P}=(-1)^{P} \frac{\| \mathbf{g}_{\mathrm{cg}, P}||}{\left|\mu_{P-1}\right|} \frac{\alpha_{P}}{\sqrt{\beta_{P}}} \mathbf{g}_{\mathrm{av}, P}
$$

so the difference between the last unnormalized CG basis and the last unormalized AV basis is the scalar

$$
c_{P}=(-1)^{P} \frac{\| \mathbf{g}_{\mathrm{cg}, P}||}{\left|\mu_{P-1}\right|} \frac{\alpha_{P}}{\sqrt{\beta_{P}}} .
$$

\section{ACKNOWLEDGMENT}

The authors would like to express their gratitudes to the anonymous reviewers for their valuable comments, especially the key result given in (22).

\section{REFERENCES}

[1] H. Krim and M. Viberg, "Two decades of array signal processing research: the parametric approach," IEEE Signal Processing Magazine, vol. 13, no. 4, pp. 67-94, 1996.

[2] R. O. Schmidt, "Multiple emitter location and signal parameter estimation," IEEE Transactions on Antennas and Propagation, vol. 34, no. 3, pp. 276-280, 1986.

[3] R. Roy and T. Kailath, "ESPRIT-estimation of signal parameters via rotational invariance techniques," IEEE Transactions on Acoustics, Speech, and Signal Processing, vol. 37, no. 7, pp. 984-995, 1989.

[4] R. Kumaresan and D. W. Tufts, "Estimating the angles of arrival of multiple plane waves," IEEE Transactions on Aerospace and Electronic Systems, vol. 19, no. 1, pp. 134-139, 1983.

[5] M. Viberg, B. Ottersten, and T. Kailath, "Detection and estimation in sensor arrays using weighted subspace fitting," IEEE Transactions on Signal Processing, vol. 39, no. 11, pp. 24362449, 1991.

[6] H. Chen, T. K. Sarkar, S. A. Dianat, and J. D. Brule, "Adaptive spectral estimation by the conjugate gradient method," IEEE Transactions on Acoustics, Speech, and Signal Processing, vol. 34, no. 2, pp. 272-284, 1986.

[7] X. Yang, T. K. Sarkar, and E. Arvas, "A survey of conjugate gradient algorithms for solution of extreme eigen-problems of a symmetric matrix," IEEE Transactions on Acoustics, Speech, and Signal Processing, vol. 37, no. 10, pp. 1550-1556, 1989. 
[8] P. S. Chang and A. N. Willson Jr., "Adaptive spectral estimation using the conjugate gradient algorithm," in Proceedings of IEEE International Conference on Acoustics, Speech, and Signal Processing (ICASSP '96), vol. 5, pp. 2979-2982, Atlanta, Ga, USA, May 1996.

[9] Z. Fu and E. M. Dowling, "Conjugate gradient eigenstructure tracking for adaptive spectral estimation," IEEE Transactions on Signal Processing, vol. 43, no. 5, pp. 1151-1160, 1995.

[10] S. Choi, T. K. Sarkar, and J. Choi, "Adaptive antenna array for direction-of-arrival estimation utilizing the conjugate gradient method," Signal Processing, vol. 45, no. 3, pp. 313-327, 1995.

[11] P. S. Chang and A. N. Willson Jr., "Conjugate gradient method for adaptive direction-of-arrival estimation of coherent signals," in Proceedings of IEEE International Conference on Acoustics, Speech, and Signal Processing (ICASSP '97), vol. 3, pp. 2281-2284, Munich, Germany, April 1997.

[12] G. H. Golub and C. F. V. Loan, Matrix Computations, Johns Hopkins University Press, Baltimore, Md, USA, 3rd edition, 1996.

[13] R. Grover, D. A. Pados, and M. J. Medley, "Super-resolution direction finding with an auxiliary-vector basis," in Digital Wireless Communications VII and Space Communication Technologies, vol. 5819 of Proceedings of SPIE, pp. 357-365, Orlando, Fla, USA, March 2005.

[14] R. Grover, D. A. Pados, and M. J. Medley, "Subspace direction finding with an auxiliary-vector basis," IEEE Transactions on Signal Processing, vol. 55, no. 2, pp. 758-763, 2007.

[15] S. Burykh and K. Abed-Meraim, "Reduced-rank adaptive filtering using Krylov subspace," EURASIP Journal on Applied Signal Processing, vol. 2002, no. 12, pp. 1387-1400, 2002.

[16] W. Chen, U. Mitra, and P. Schniter, "On the equivalence of three reduced rank linear estimators with applications to DSCDMA," IEEE Transactions on Information Theory, vol. 48, no. 9, pp. 2609-2614, 2002.

[17] G. Xu and T. Kailath, "Fast subspace decomposition," IEEE Transactions on Signal Processing, vol. 42, no. 3, pp. 539-551, 1994.

[18] D. A. Pados and S. N. Batalama, "Joint space-time auxiliaryvector filtering for DS/CDMA systems with antenna arrays," IEEE Transactions on Communications, vol. 47, no. 9, pp. 14061415, 1999.

[19] D. A. Pados and G. N. Karystinos, "An iterative algorithm for the computation of the MVDR filter," IEEE Transactions on Signal Processing, vol. 49, no. 2, pp. 290-300, 2001.

[20] Q. T. Zhang, "Probability of resolution of the MUSIC algorithm," IEEE Transactions on Signal Processing, vol. 43, no. 4, pp. 978-987, 1995.

[21] S. U. Pillai and B. H. Kwon, "Forward/backward spatial smoothing techniques for coherent signal identification," IEEE Transactions on Acoustics, Speech, and Signal Processing, vol. 37, no. 1, pp. 8-15, 1989.

[22] H. Qian and S. N. Batalama, "Data record-based criteria for the selection of an auxiliary vector estimator of the MMSE/MVDR filter," IEEE Transactions on Communications, vol. 51, no. 10, pp. 1700-1708, 2003.

[23] D. Segovia-Vargas, F. Iñigo, and M. Sierra-Pérez, "Generalized eigenspace beamformer based on CG-Lanczos algorithm," IEEE Transactions on Antennas and Propagation, vol. 51, no. 8, pp. 2146-2154, 2003.
Hichem Semira was born on December 21, 1973 in Constantine, Algeria. He received the B.Eng. degree in electronics in 1996 and the Magistère degree in signal processing in 1999 both from Constantine University (Algeria). He is now working towards Ph.D. degree in the Department of Electronics at Annaba University (Algeria). His research interests are in signal processing for communications, and array processing.

Hocine Belkacemi was born in Biskra, Algeria. He received the engineering degree in electronics from the Institut $\mathrm{Na}$ tional d'Electricité et d'Electronique (INELEC), Boumerdes, Algeria, in 1996, the Magistère degree in electronic systems from École Militaire Polytechnique, Bordj El Bahri, Algeria, in 2000, the M.S. (D.E.A.) degree in control and signal processing and the Ph.D. degree in signal processing both from Uni-

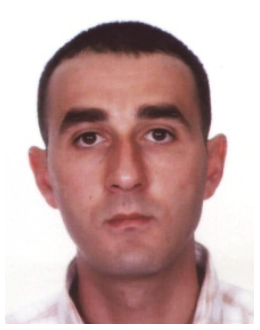
versité de Paris-Sud XI, Orsay, France, in 2002 and 2006, respectively. He is currently an Assistant Teacher with the radio communication group at the Conservatoire National des Arts et Métiers CNAM, Paris, France. His research interests include array signal processing with application to radar and communications, adaptive filtering, non-Gaussian signal detection and estimation.

Sylvie Marcos received the engineer degree from the Ecole Centrale de Paris (1984) and both the Doctorate (1987) and the Habilitation (1995) degrees from Université de ParisSud XI, Orsay, France. She is Directeur de Recherche at the National Center for Scientific Research (CNRS) and works in Laboratoire des Signaux et Systèmes (LSS) at Supélec, Gif-sur-Yvette, France. Her main research interests are presently array pro-

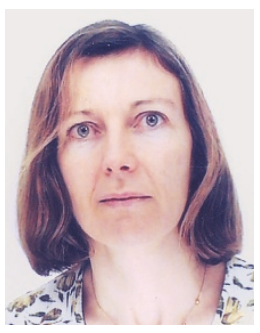
cessing, spatio-temporal signal processing (STAP) with applications in radar and radio communications, adaptive filtering, linear and nonlinear equalization and multiuser detection for CDMA systems. 